\title{
On the consistency of intramodal intensity matching in olfaction*
}

\author{
LEIF T. SVENSSON† \\ Department of Environmental Hygiene \\ National Institute of Public Health, S-104 01 Stockholm, Sweden
}

and

\author{
THOMAS LINDVALL†† \\ Department of Environmental Hygiene \\ Karolinska Institute and Swedish Environment Protection Board, S-104 01 Stockholm, Sweden
}

Magnitude matchings of odor intensity were obtained for three chemical compounds (hydrogen sulfide, pyridine, and dimethyl disulfide) in four experiments. An equal-sensation function in the form of a power function described the data well. This equation can be written neglecting the multiplicative constant,

$$
\phi_{\mathrm{i}}=\phi_{\mathrm{k}}{ }^{\mathrm{b}} \mathrm{ik},
$$

where $i$ and $k$ represent two different odorants. The exponent $b_{i k}$, where the first index stands for matching continuum and the second index for reference continuum, was fairly well predicted according to the formulae,

$$
\begin{aligned}
& b_{i k}=b_{i j}\left[b_{j k}+\frac{1}{b_{k j}}\right] \frac{1}{2} \\
& b_{i k}=\left[\frac{1}{b_{k i}}+\frac{b_{j k}}{b_{j i}}\right] \frac{1}{2}
\end{aligned}
$$

where $i, j$, and $k$ are three different odorants. The results validate the equal-sensation function and indicate implicitly that intensity matchings between different intramodal continua are approximately transitive and symmetric with respect to odor.

Modern psychophysical methods of analysis, such as magnitude matching procedures, are being increasingly used in basic as well as in applied odor research. It would be desirable to make the scale values obtained comparable from one laboratory to another by including a standard odorant in all scaling experiments. Such a standard would increase comparability of experimental findings and facilitate progress in this research area. For example, hydrogen sulfide could be a satisfactory standard odor in environmental health research. The authors also have

\footnotetext{
*The investigation was supported by grants from the Swedish Environment Protection Board and the Folksam Research Board and by a grant to B. Berglund from the Swedish Council for Social Science Research. Thanks go to P.-O. Olsson, S. Pettersson, and G. Rosén for assistance with the experiments.

How at the Psychological Laboratories, University of Stockholm, Box 6706, S-113 85 Stockholm, Sweden.

††Reprint requests should be sent to: Thomas Lindvall, Department of Environmental Hygiene, Karolinska Institute, S-104 01 Stockholm, Sweden.
}

advocated the necessity of a validation of the exponent in the equal-sensation function by the use of one experimental setting with all odorants being matched (cf. Lindvall \& Svensson, 1974). In basic research, a similar standpoint is expressed by Cain (1971). He suggests n-butanol as a possible standard odorant. The validation problem is approached in the present investigation by studying the consistency of the equal-sensation function obtained in intramodal matching experiments with three odorants.

Let us first consider the basis of the equal-sensation function. Assuming that the power function, $\psi=$ $\mathrm{a} \varnothing \mathrm{n}$, adequately describes the relation between perceptual and physical continua for the attribute's intensity or unpleasantness, we may write the following for two different continua, neglecting the multiplicative constants

$$
\psi_{1}=\phi_{1}{ }^{\mathrm{m}} \text { and } \psi_{2}=\phi_{2}^{\mathrm{n}}
$$


In intermodality, as well as in intramodality magnitude matching, the Ss are instructed to equate $\psi_{1}$ and $\psi_{2}$ at different stimulation levels. That is, the equal-sensation function will have the form

$$
\phi_{1}^{\mathrm{m}}=\phi_{2}^{\mathrm{n}} \text {. }
$$

Solving for $\varnothing_{1}$ and $\varnothing_{2}$ in Eq. 2, we obtain

$$
\phi_{1}=\phi_{2}{ }^{b_{12}}, \text { where } b_{12}=n / m
$$

and

$$
\phi_{2}=\phi_{1}{ }^{b_{21}} \text {, where } b_{21}=\mathrm{m} / \mathrm{n} \text {. }
$$

The predicted functions have been obtained in experiments on intermodality matching of subjective intensity for several pairs of modalities (see, e.g., Stevens, 1960). They have also been verified experimentally for intramodality matching in audition (see, e.g., Stevens, 1966), for heterochromatic matching of saturation of a hue to saturation of another hue (Indow \& Stevens, 1966), for vibrotactile stimuli (Stevens, 1968), and for matching of linear aliphatic alcohols (Cain, 1969).

Lindvall and Svensson (1974) showed that the functional relationship (Eqs. 3 and 4) earlier found in intermodality matching and within some other modalities also applies to intramodality matching in olfaction, at least with the attribute of unpleasantness.

\section{Transitivity, Symmetry, and Reflexivity}

It will be possible to use a standard odorant as a relative measure of malodorous pollutants only if the matchings between pairs of odors on different levels of intensity are transitive, symmetric, and reflexive. In formal language, we can write this in the following way.

Let $i, j$, and $k$ be three different continua and $\varnothing_{i}$, $\varnothing_{j}$, and $\varnothing_{k}$ physical intensities of these continua. Let $M$ be the matching relation. Then we have:

Transitivity: If $\varnothing_{\mathrm{i}} \mathrm{M} \varnothing_{\mathrm{j}}$ and $\varnothing_{\mathrm{j}} \mathrm{M} \varnothing_{\mathrm{k}}$, then $\varnothing_{\mathrm{i}} \mathrm{M} \varnothing_{\mathrm{k}}$

\section{Symmetry: If $\varnothing_{\mathrm{i}} \mathrm{M} \varnothing_{\mathrm{j}}$, then $\varnothing_{\mathrm{j}} \mathrm{M} \varnothing_{\mathrm{i}}$}

$$
\text { Reflexivity: If } \varnothing_{\mathrm{i}} \mathrm{M} \varnothing_{\mathrm{i}} \text {, then } \varnothing_{\mathrm{i}}=\varnothing_{\mathrm{i}}
$$

There are certain given relations between exponents if the relations above are valid. Let us rewrite the equal-sensation function (Eqs. 3 and 4 ) in the following way:

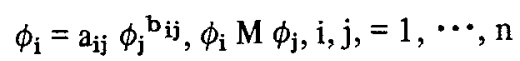

where the first index (i) represents the matching continuum and the second (j) the reference continuum. Then the following equations should hold, neglecting the multiplicative constants $a_{i k}, a_{i j}$, ajk:

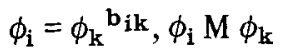

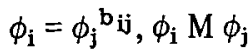

$$
\begin{aligned}
& \phi_{j}=\phi_{k}^{\mathbf{b}_{j k}}, \phi_{j} M \phi_{k}
\end{aligned}
$$

Equations 7 and 8 give

$$
\begin{aligned}
& \phi_{\mathrm{i}}=\left[\phi_{\mathbf{k}}^{\mathbf{b}_{\mathrm{jk}}}\right]^{\mathbf{b}_{\mathrm{ij}}} \\
& \phi_{\mathrm{i}}=\phi_{\mathrm{k}}^{\mathbf{b}_{\mathrm{ij}} \mathbf{b}_{\mathrm{jk}} .}
\end{aligned}
$$

From Eqs. 6 and 9, we have

$$
b_{i j} b_{j k}=b_{i k} \text {, if } \phi_{i} M \phi_{j} \text { and } \phi_{j} M \phi_{k} \text { then } \phi_{i} M \phi_{k} \text {. }
$$

Equation 10 is a necessary condition for the equalsensation function to be transitive. Generalizing Eqs. 3 and 4 in accordance with the conventions and comparing exponents, we get,

$$
\mathrm{b}_{\mathrm{ij}} \mathrm{b}_{\mathrm{ji}}=1 \text {, if } \phi_{\mathbf{i}} \mathrm{M} \phi_{\mathrm{j}} \text { then } \phi_{\mathrm{j}} \mathrm{M} \phi_{\mathbf{i}} \text {. }
$$

Equation 11 is a necessary condition for the equalsensation function to be symmetric.

Stimuli on a continuum matched to different stimuli on the same continuum would give the exponent a value of 1 , i.e.,

$$
b_{i \mathrm{i}}=1 \text {, if } \phi_{\mathrm{i}} \mathrm{M} \phi_{\mathrm{i}} \text { then } \phi_{\mathrm{i}}=\phi_{\mathrm{i}} .
$$

Equation 12 is a necessary condition for the equalsensation function to be reflexive.

\section{PROBLEM}

The present paper is specifically concerned with the transitivity and symmetry criteria for intramodal magnitude matching of odor intensity. In order to test these two criteria, an experiment was designed which

Table 1

Exponents in the Equal-Sensation Function that are Possible to Estimate Through Pairwise Magnitude Matchings of Three Continua

\begin{tabular}{cccc}
\hline $\begin{array}{c}\text { Match- } \\
\text { ing } \\
\text { Continua }\end{array}$ & \multicolumn{3}{c}{ Reference Continua } \\
\cline { 2 - 4 } & $\phi_{1}$ & $\phi_{2}$ & $\phi_{3}$ \\
\hline$\phi_{1}$ & $\mathrm{~b}_{1_{2}}$ & $\mathrm{~b}_{12}$ & $\mathrm{~b}_{13}$ \\
$\phi_{2}$ & $\mathrm{~b}_{21}$ & $\mathrm{~b}_{22}$ & $\mathrm{~b}_{23}$ \\
$\phi_{3}$ & $\mathrm{~b}_{31}$ & $\mathrm{~b}_{32}$ & $\mathrm{~b}_{33}$ \\
\hline
\end{tabular}


Table 2

Exponents Predicted from the Transitivity Criterion

\begin{tabular}{cccc}
\hline & \multicolumn{3}{c}{ Reference Continua } \\
\cline { 2 - 4 } Matching & $\phi_{1}$ & $\phi_{2}$ & $\phi_{3}$ \\
\hline$\phi_{1}$ & 1 & $\mathrm{~b}_{13} \mathrm{~b}_{32}$ & $\mathrm{~b}_{12} \mathrm{~b}_{23}$ \\
$\phi_{2}$ & $\mathrm{~b}_{23} \mathrm{~b}_{31}$ & 1 & $\mathrm{~b}_{21} \mathrm{~b}_{13}$ \\
$\phi_{3}$ & $\mathrm{~b}_{32} \mathrm{~b}_{21}$ & $\mathrm{~b}_{31} \mathrm{~b}_{12}$ & 1 \\
\hline
\end{tabular}

included three different odorants. In Table 1 is shown a matrix with the exponents in the equal-sensation function that are possible to estimate through all possible pairwise magnitude matchings of the three odorants. Row head shows the matching continua, $\varnothing_{1}, \varnothing_{2}$, and $\varnothing_{3}$. Column head shows the reference continua, $\varnothing_{1}, \varnothing_{2}$, and $\varnothing_{3}$. Cell entries show all matching exponents possible to estimate.

If the transitivity criterion holds, the predicted exponent matrix presented in Table 2 will be valid. In the diagonal, the values of 1 indicate that the reflexivity criterion holds.

In the same way, exponents can be predicted from the symmetry criterion, as presented in Table 3 . Here also, the values of 1 in the diagonal indicate reflexivity.

Let us call the empirical exponent matrix $B$ (Table 1). In the same way, we have two matrices predicted from the empirical exponent matrix, B, if the transitivity and symmetry criteria hold. Let them be $B_{t}$ (Table 2 ) and $B_{S}$ (Table 3), respectively.

From $B, B_{t}$, and $B_{S}$, we can derive two more predicted matrices. These are valid if both the transitivity criterion and symmetry criterion hold. Let them be $B_{\text {ts }}$ and $B_{\text {ts. }}^{\prime}$ Matrices $B_{t s}$ and $B^{\prime}$ ts are used in the following to predict one exponent from three others.

$$
\begin{aligned}
& B_{t s}=\left[\begin{array}{ccc}
1 & b_{13} / b_{23} & b_{12} / b_{32} \\
b_{23} / b_{13} & 1 & b_{21} / b_{31} \\
b_{32} / b_{12} & b_{31} / b_{21} & 1
\end{array}\right] \\
& B_{t s}^{\prime}=\left[\begin{array}{ccc}
1 & b_{32} / b_{31} & b_{23} / b_{21} \\
b_{31} / b_{32} & 1 & b_{13} / b_{12} \\
b_{21} / b_{23} & b_{12} / b_{13} & 1
\end{array}\right]
\end{aligned}
$$

The odorants used in the present experiment were matched with respect to intensity to estimate the exponents, $b_{21}, b_{31}, b_{13}$, and $b_{23}$. A necessary requirement for this procedure is that the equalsensation function can be demonstrated to be of the general form shown in Eq. 5. First, from $B, B_{t}$, and $\mathrm{B}_{\mathrm{ts}}$, we have,
Table 3

Exponents Predicted from the Symmetry Criterion

\begin{tabular}{cccc}
\hline & \multicolumn{3}{c}{ Reference Continua } \\
\cline { 2 - 4 } Matching & $\phi_{1}$ & $\phi_{2}$ & $\phi_{3}$ \\
\hline$\phi_{1}$ & 1 & $1 / \mathrm{b}_{21}$ & $1 / \mathrm{b}_{31}$ \\
$\phi_{2}$ & $1 / \mathrm{b}_{12}$ & 1 & $1 / \mathrm{b}_{32}$ \\
$\phi_{3}$ & $1 / \mathrm{b}_{13}$ & $1 / \mathrm{b}_{23}$ & 1 \\
\hline
\end{tabular}

$b_{21}=b_{23} b_{31}=b_{23} / b_{13} ; b_{21}=b_{23}\left[b_{31}+\frac{1}{b_{13}}\right] \frac{1}{2}$
$b_{23}=b_{21} b_{13}=b_{21} / b_{31} ; b_{23}=b_{21}\left[b_{13}+\frac{1}{b_{31}}\right] \frac{1}{2}$

that is, in general terminology for three continua, $i, j$, and $\mathbf{k}$,

$$
b_{i k}=b_{i j}\left[b_{j k}+\frac{1}{b_{k j}}\right] \frac{1}{2}
$$

Second, from $B, B_{S}$, and $B^{\prime}$ ts, we have,

$$
\begin{aligned}
& b_{13}=1 / b_{31}=b_{23} / b_{21} ; \quad b_{13}=\left[\frac{1}{b_{31}}+\frac{b_{23}}{b_{21}}\right] \frac{1}{2} \\
& b_{31}=1 / b_{13}=b_{21} / b_{23} ; b_{31}=\left[\frac{1}{b_{13}}+\frac{b_{21}}{b_{23}}\right] \frac{1}{2}
\end{aligned}
$$

that is, in general, for three continua, $i, j$, and $k$,

$$
b_{i k}=\left[\frac{1}{b_{k i}}+\frac{b_{j k}}{b_{j i}}\right] \frac{1}{2} .
$$

The hypothesis is that these relations are valid. In fact, they are necessary conditions for the equalsensation function to be transitive and symmetric. However, they are not sufficient. In order to test necessary and sufficient criteria, the "true" function must be known.

\section{THE EXPERIMENTS}

\section{Subjects}

Two women and two men, aged 20-30 years, participated in the experiment. They were students without any previous experience of psychological experiments.

\section{Stimuli}

The odorous substances were hydrogen sulfide $\left(\mathrm{H}_{2} \mathrm{~S}\right.$; Matheson, technical grade), pyridine (Pyr; Mallinckrodt, analytical reagent), and dimethyl disulfide (DMDS; Eastman, 97.6\% GLC).

The hydrogen sulfide was kept in gas form in tubes. The gas was taken directly from the tubes and led into the dosage system. Pyridine and dimethyl disulfide were kept in liquid form in impinger tubes. The tubes were submerged in a thermostatically 
controlled water bath $\left(16^{\circ} \mathrm{C}\right)$, and a continuous stream of nitrogen was swept over the surface of the substances. This flow was regulated so as to obtain a continuous stream of nitrogen laden with the odorous gas.

\section{Chemical Analysis}

Analysis of hydrogen sulfide was carried out once for every $S$ and session by employing the gel technique method described by Broström and Brosset (1969). Samples were taken immediately in front of the dosing capillaries.

Pyridine and dimethyl disulfide were analyzed with a gas chromatograph (Aerograph $204 \mathrm{~B}$ ). The analyses were run using $5 \%$ Carbowax $20 \mathrm{M} /$ chromosorb $\mathrm{W}$ as a column at $100^{\circ} \mathrm{C}$ and flame ionization detector. The gas chromatograph was calibrated with a dilution of pyridine in water and a dilution of dimethyl disulfide in carbontetrachloride, respectively.

\section{Experimental Equipment}

The laboratory is constructed as a mobile unit for sensory evaluation of odorous air pollutants. The olfactometer involves a well-defined dynamic flow of gas which permits stable exposure concentrations. rapid changes in concentration, and fairly natural respiratory conditions (Lindvall, 1970). The equipment consists essentially of a ventilated and air-conditioned test chamber, in which two Teflon-coated exposure hoods have been installed. The odorant gas is mixed with purified air in fixed dilutions and added to the flow of air into the hoods by means of electromagnetically operated steel and Teflon cannulae of varying bore. A special waiting room for the $S s$, with ventilation comparable to the actual test procedure, provides optimal adaptation. By using suitable filter equipment (active charcoal filters and particle filters), it is possible to carry out evaluations with a minimum of contaminations in the laboratory air.

\section{Process Control}

In order to obtain a precise control of the stimulation during the matchings, a new method of successive approximations with automatic equipment was developed (Svensson \& Szczygiel, 1974). The reference stimulus is presented in one of the hoods and the matching stimulus in the other. The Ss have to decide whether (1) the odor in the left hood is the more intense, (2) the odors in the two hoods are equally intense, or indistinguishable, or (3) the odor in the right hood is the more intense.

The responses are fed into a logic unit by an electrical signal system. The information is then processed and a new matching stimulus is automatically initiated, according to an algorithm that bisects intervals up or down in successive trials.

The $S$ can make a decision in four trials at most. As soon as the $S$ signals the second response alternative above, no further matching stimulus is presented, the logic unit stops, and the result is visualized on a panel to the $E$. If perceived equality is not attained at Trial 4 , the result will be reported as being more or less than the nearest stimuli chosen. This strategy is optimal, with four binary decisions to find 1 interval out of 16 possible (see further, Svensson \& Szcyzygiel, 1974).

The judgment situation is easily understood, even by naive Ss, and the strategy is the same for all. Distortion effects due to cognitive, motivational, and learning factors are minimized.

Table 4

Absolute Odor Thresholds for Three Odorants Expressed in $-\log (\mathrm{mg} / \mathrm{1})$

\begin{tabular}{lccccc}
\hline & \multicolumn{5}{c}{ Subject } \\
\cline { 2 - 5 } Substance & A & B & C & D & Mean \\
\hline $\mathrm{H}_{2} \mathrm{~S}$ & 5.99 & 5.57 & 5.52 & 5.65 & 5.68 \\
Pyridine & 3.91 & 3.93 & 3.77 & 3.98 & 3.90 \\
DMDS & 4.59 & 4.46 & 4.29 & 4.31 & 4.41 \\
\hline
\end{tabular}

\section{Procedure}

In order to validate the equal-sensation function, four experimental combinations, out of nine possible, were studied. Pyridine and DMDS, respectively, were matched to $\mathrm{H}_{2} \mathrm{~S}$, and $\mathrm{H}_{2} \mathrm{~S}$ and pyridine, respectively, were matched to DMDS. Combinations $\mathrm{H}_{2} \mathrm{~S}-\mathrm{H}_{2} \mathrm{~S}$, Pyr-Pyr, and DMDS-DMDS were not considered, as these are less interesting and possess a high possibility of giving the exponent 1 in the power function.

The method of magnitude matching was used. In the left hood, Ss were exposed to 5 concentrations of the reference odorant ordered in a geometrical progression. In the right hood, the Ss could be presented with any of 15 different concentrations of the matching odorant, also ordered in a geometrical series. The five reference stimuli were presented in random ordet in every session.

In a pilot study, 10 absolute thresholds were obtained by a modified method of limits for every $\mathrm{S}$ and for every odorant (cf. Lindvall, 1970). In Table 4, arithmetic means of individual thresholds are presented. They are the mean of 10 determinations for each $\mathrm{S}$. Arithmetic group mean values are also tabulated.

The reference odorants in $\mathrm{mg} /$ liter were chosen as

$$
10^{t_{r}+0.5 k}
$$

where $t_{r}$ is $-\log$ threshold concentration and $k=1, \ldots 5$ for the 5 reference stimuli. The 15 matching stimuli were chosen in accordance with the expression,

$$
10^{t_{m}+0.25 k}
$$

where $t_{m}$ is - $\log$ threshold concentration in $\mathrm{mg} /$ liter and $\mathrm{k}=0, \ldots$, 14 for the 15 matching stimuli. By this selection, the range of the matching odorant is intended to cover the range of the reference odorant and thus minimize possible category effects.

A green light inside the hood flashed for $8 \mathrm{sec}$. Within this time, the $S$ had to make a magnitude match by first taking a sniff in one of the hoods and then quickly turning to the other for another sniff. Between trials, the $S$ had to wait for $18 \mathrm{sec}$. The Ss were thoroughly instructed and supervised in this task.

The Ss participated for 4 days each, and two experimental combinations were run every day. The sessions were run according to an ABBA design. The order in which the $S$ was to smell the stimuli in the two hoods was also balanced-first the reference stimulus and then the matching stimulus, or the opposite.

\section{RESULTS AND DISCUSSION}

\section{Equal-Sensation Functions}

In Fig. 1, the 16 individual equal-sensation plots are shown, with 4 plots for each experimental combination and each $S$. The concentrations are expressed as $\log \left(\mathrm{S} / \mathrm{S}_{\mathrm{O}}\right)$, where the numerator $(\mathrm{S})$ is the odor intensity in $\mathrm{mg} / \mathrm{liter}$ and the denominator $\left(\mathrm{S}_{\mathrm{O}}\right)$ is the absolute threshold in $\mathrm{mg} /$ liter. Each point in the plots represents the arithmetic mean of 16 matchings. The trends in the equal-sensation plots are approximately linear. Straight lines are therefore fitted to the data by means of the method of least squares. Thus, in linear coordinates, a power function fits to the data well. This result is in accordance with what Lindvall and Sxensson (1974) found for intramodal unpleasantness matching in olfaction and with what earlier has been demonstrated in intramodal intensity matching for several modalities (see, e.g., Stevens, 1966; Indow \& Stevens, 1966; Stevens, 1968; Cain, 1969). 

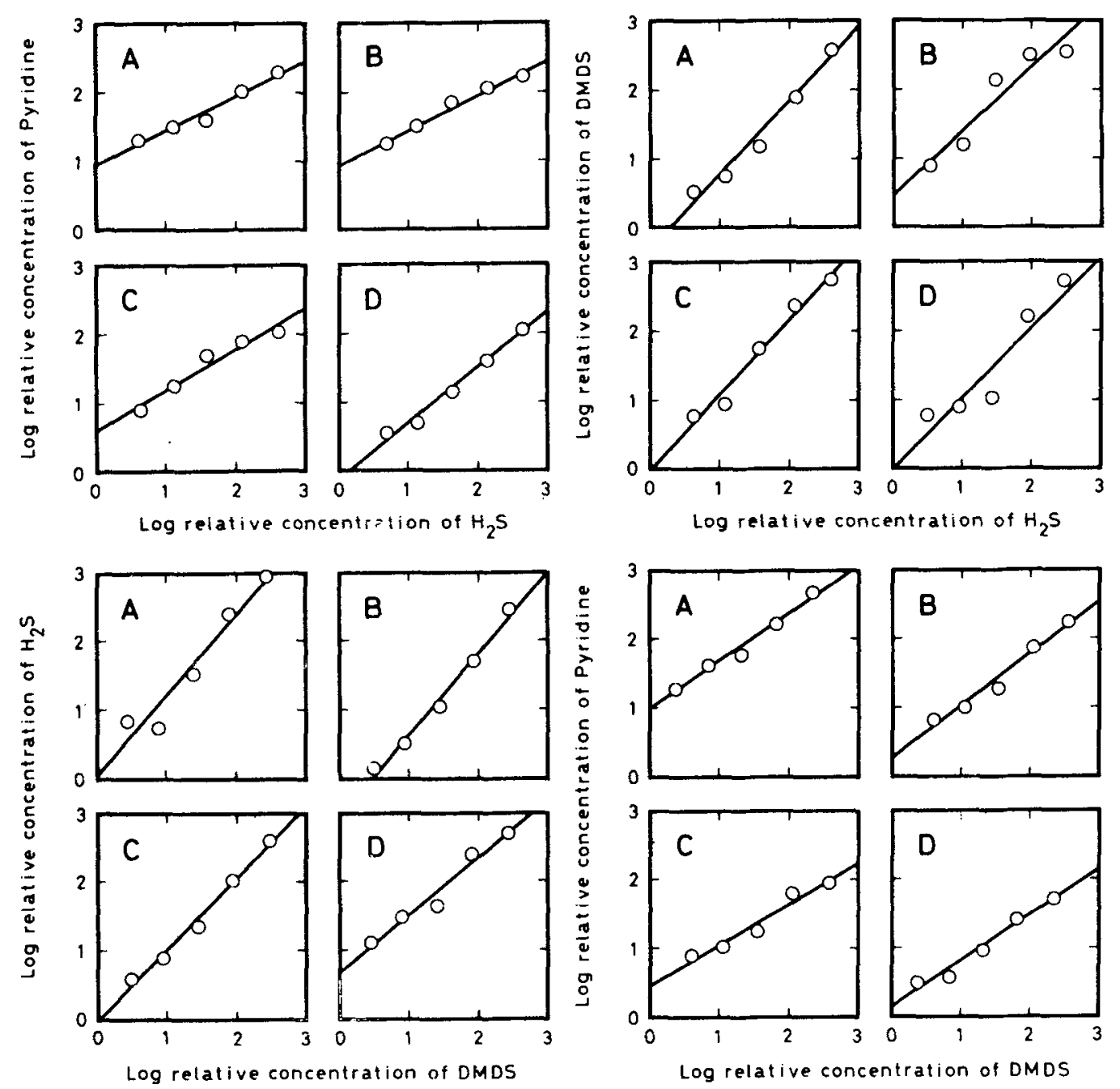

Fig. 1. Equal-sensation functions in log-log coordinates obtained for four Ss (A-D) and four experiments: I-pyridine matched to hydrogen sulfide, II-dimethyl disulfide matched to hydrogen sulfide, III-hydrogen sulfide matched to dimethyl disulfide, and IV - pyridine matched to dimethyl disulfide. The straight lines are fitted by the method of least squares. The concentrations are expressed relative to absolute thresholds.

In Fig. 2, four matching plots are shown for group data, one for each experimental combination. In the $\log -\log$ plots, the linearity is striking.

It is a well-known fact that olfactory adaptation is very rapid (Ekman, Berglund, Berglund, \& Lindvall, 1967) and that absolute thresholds are influenced by a large number of variables (e.g., Lindvall, 1970). As matchings in olfaction cannot be made simultaneously, but only successively, the time for making a complete match can be considerable with traditional production methods. The perceptual functioning may be unnecessarily influenced by, for example, habituation and diurnal variation (see, e.g., Engen, 1973). It is probable that the time-saving method of successive approximations (Svensson \& Szczygiel, 1974) and the well-controlled design of the present experiment have been of importance for the demonstrated findings. Magnitude matching with successive approximations is therefore regarded by the authors as a valuable complement to modern psychophysical scaling methods in perceptual research.

\section{Empirical vs Predicted Exponents}

Table 5 shows schematically the empirical exponents and the exponents predicted from the empirical ones according to Eqs. 13, 14, 16, and 17. The good fit of data to these equations is evident from Table 6. The expressions "Pred(1)" and "Pred(2)" stand for the two possible predictions of the exponent in a single experiment that can be made from the exponents empirically obtained in the three remaining experiments. The arithmetic mean of these two predictions is called "Mean pred."

From Table 6, it can be seen that the data from Ss $\mathrm{C}$ and $\mathrm{D}$ fit the formulae comparatively well, while 
Table 5

Exponents of the EqualSensation Function Possible to Predict and Empirical Exponents in the Four Experimental Conditions

\begin{tabular}{|c|c|c|c|c|}
\hline \multirow{2}{*}{$\begin{array}{c}\text { Experi- } \\
\text { ment }\end{array}$} & \multicolumn{4}{|c|}{ Exponent } \\
\hline & Pred 1 & Pred 2 & Mean Pred & Emp \\
\hline $\begin{array}{l}\text { I } \\
\text { II } \\
\text { III } \\
\text { IV }\end{array}$ & $\begin{array}{l}b_{23} b_{31} \\
1 / b_{13} \\
1 / b_{31} \\
b_{21} b_{13}\end{array}$ & $\begin{array}{l}\mathrm{b}_{23} / \mathrm{b}_{13} \\
\mathrm{~b}_{21} / \mathrm{b}_{23} \\
\mathrm{~b}_{23} / \mathrm{b}_{21} \\
\mathrm{~b}_{21} / \mathrm{b}_{31}\end{array}$ & $\begin{array}{l}\mathrm{b}_{23}\left[\mathrm{~b}_{31}+\left(1 / \mathrm{b}_{13}\right)\right]^{1 / 2} \\
{\left[\left(1 / \mathrm{b}_{13}\right)+\left(\mathrm{b}_{21} / \mathrm{b}_{23}\right)\right]^{1 / 2}} \\
{\left[\left(1 / \mathrm{b}_{31}\right)+\left(\mathrm{b}_{23} / \mathrm{b}_{21}\right)\right]^{1 / 2}} \\
\mathrm{~b}_{21}\left[\mathrm{~b}_{13}+\left(1 / \mathrm{b}_{31}\right)\right]^{1 / 2}\end{array}$ & $\begin{array}{l}b_{21} \\
b_{31} \\
b_{13} \\
b_{23}\end{array}$ \\
\hline
\end{tabular}

those from the other two Ss, who were women, display a greater dispersion. From earlier experiments, it has been argued that olfactory sensitivity changes during the ovulatory cycle (Le Magnen, 1950). The present experiment lasted for more than 2 weeks, and thus the ovulatory cycle cannot be excluded as a candidate for interference with perceptions. To be revealed, the causes of dispersion need further experimentation. The data are summarized in Fig. 3, where empirical exponents are plotted against predicted ones.

The open symbols represent different Ss, and the filled circles, group data. The diagonal in the figure stands for perfect fit of data to theory. The individual differences in the data of Table 6 are again illustrated in this plot. Although the scatter of the individual data is considerable, the group data and the data from one of the Ss are well centered around the diagonal. From the data in Table 6, it can hardly be
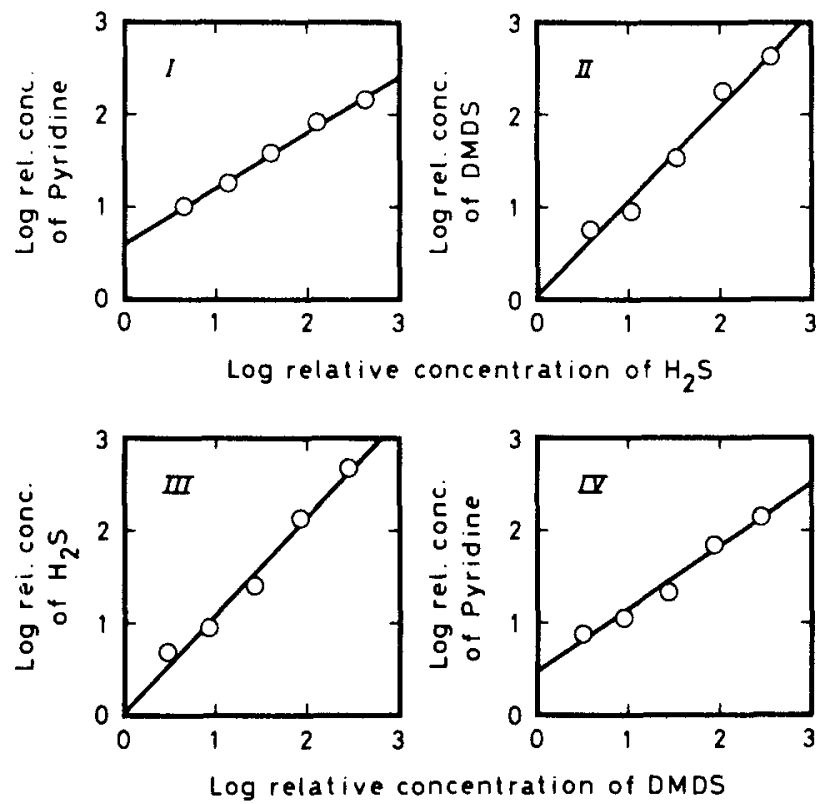

Fig. 2. Equal-sensation functions in log-log coordinates obtained for group data in the four experiments: I-pyridine matched to hydrogen sulfide, II-dimethyl disulfide matched to hydrogen sulfide, III-hydrogen sulfide matched to dimethyl disulfide, and IV-pyridine matched to dimethyl disulfide. The straight lines are fitted by means of the method of least squares. The concentrations are expressed relative to absolute thresholds for the individual Ss. said that the two hypotheses of symmetry and transitivity are verified separately (independently) from predictions (Eq. 1) in Experiments II, III, and I, IV, respectively. But the transitivity and symmetry criteria are implicit in the mean predicted exponents (Eqs. 13, 14, 16, and 17). Hence, the exponents in the equal-sensation function seem to be consistent as a first approximation.

It is important to note that the relationship between exponents obtained in magnitude estimation and magnitude matching experiments (Eqs. 1 and 2) shown by Stevens (1960; see also Cain, 1969) is not considered here. The deviation from Eqs. 15 and 18 may partly depend on regression effects in the psychophysical judgments similar to the effects shown by Stevens and Greenbaum (1966) when vibration applied to the fingertip was matched to loudness of a noise.

Table 6

Empirical Exponents of the Equal-Sensation Function and Exponents Predicted from One or Two Other Empirical Exponents in Experiments I-IV* (cf. Table 4)

\begin{tabular}{cccccc}
\hline \multirow{2}{*}{$\begin{array}{c}\text { Experi- } \\
\text { ment }\end{array}$} & S & \multicolumn{4}{c}{ Exponent } \\
\cline { 3 - 6 } I & Pred 1 & Pred 2 & $\overline{\text { X }}$ Pred & Emp \\
\hline & A & 0.74 & 0.58 & 0.66 & 0.50 \\
& B & $\mathbf{0 . 7 0}$ & 0.64 & 0.67 & 0.51 \\
& C & 0.64 & 0.57 & 0.61 & 0.59 \\
& D & 0.68 & 0.73 & 0.71 & 0.80 \\
& Mean & 0.69 & 0.64 & 0.67 & 0.70 \\
II & A & 0.85 & 0.72 & 0.79 & 1.07 \\
& B & 0.85 & 0.68 & 0.77 & 0.93 \\
& C & 0.96 & 1.01 & 0.99 & 1.09 \\
& D & 1.20 & 1.20 & 1.20 & 1.02 \\
& Mean & 0.94 & 0.89 & 0.92 & 1.03 \\
III & A & 0.94 & 1.39 & 1.17 & 1.18 \\
& B & 1.08 & 1.47 & 1.28 & 1.18 \\
& C & 0.92 & 1.00 & 0.96 & 1.04 \\
& D & 0.98 & 0.84 & 0.91 & 0.83 \\
& Mean & 0.98 & 1.13 & 1.06 & 1.06 \\
IV & A & 0.59 & 0.47 & 0.53 & 0.69 \\
& B & 0.60 & 0.55 & 0.58 & 0.76 \\
& C & 0.61 & 0.54 & 0.58 & 0.59 \\
& D & 0.67 & 0.78 & 0.73 & 0.67 \\
& Mean & 0.64 & 0.58 & 0.61 & 0.68 \\
\hline
\end{tabular}

*Pyridine (I) and DMDS (II) matched to $\mathrm{H}_{2} \mathrm{~S} ; \mathrm{H}_{2} \mathrm{~S}$ (III) and pyridine (IV) matched to DMDS. 


\section{Relevance for Environmental Psychology}

The present results indicate that a standard odorant may be a reliable tool in future environmental health studies of sensory effects. Different pollutants may hence be compared with one another, according to Eqs. 15 and 18, when matched to a standard odorant. That is to say, knowledge of the exponents obtained in matching experiments with the standard odorant and one pollutant at a time will allow prediction of the exponent that would be obtained when two different pollutants are matched directly to each other. In order to obtain psychological scales for different odorous air pollutants, the standard odorant has to be scaled by, for example, magnitude estimation. Scale transformations can then be performed in order to find the psychological scales for the different air pollutants. Of course, this two-step technique needs further investigations to test its general usefulness.

\section{Future Research}

The results of this experiment point towards a consistent equal-sensation function regarding the exponent. This is true both for individual data and group data. By consistency, here, is meant the joint fulfillment of the transitivity and symmetry criteria defined in the introduction and the statement of problem. The defined consistency is, however, just one necessary condition for the equal-sensation function to be consistent from a measurement theoretical stand point.

Future research must take into account parameters other than the exponent. Some functional relationship between the multiplicative constant and the exponent could give interesting interpretative meaning to the function and reduce the number of parameters. If such a relationship can be found, it will be easier to validate the equal-sensation function as a whole, which certainly would influence the development of a reliable measurement system for odors in environmental psychology. Such experiments are in progress.

\section{REFERENCES}

Broström, C. E., \& Brosset, C. A method for simultaneous determination of hydrogen sulfide and sulfur dioxide in flue gases. Atmospheric Environment, 1969, 3, 407-416.

CaIN, W. S. Odor intensity: Differences in the exponent of the psychophysical function. Perception \& Psychophysics, 1969, 6, 349-354.

CArN, W. S. Physicochemical, characteristics and supraliminal odor intensity: Reply to Mitchell. Perception \& Psychophysics. 1971, 9, 478-479.

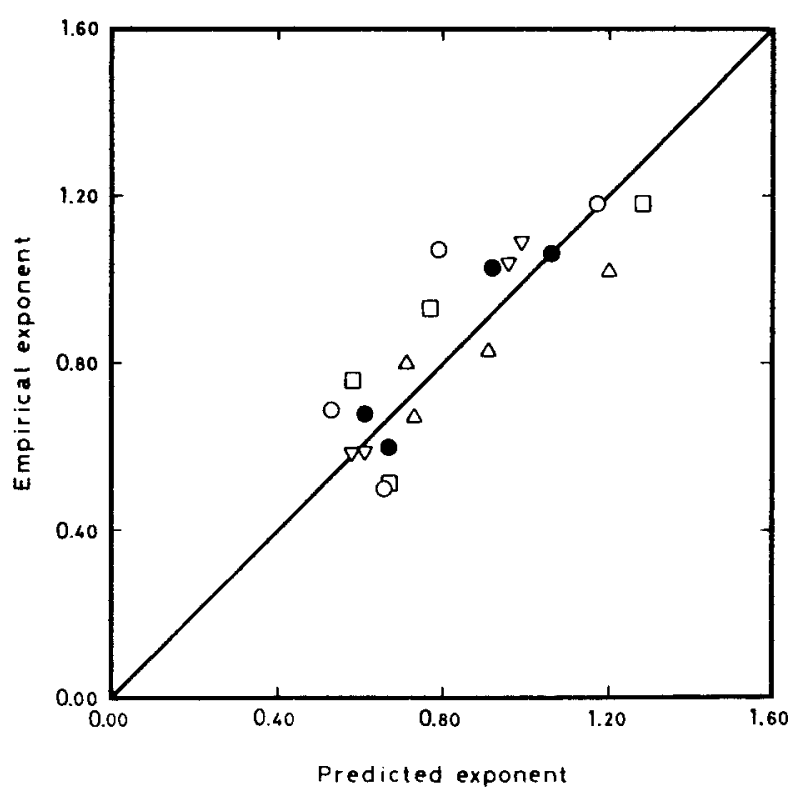

Fig. 3. Empirical exponents of the equal-sensation function plotted against predicted exponents according to Tables 5 and 6 . The different open symbols stand for different $S s$, and the filled circles stand for group data.

Engen, T. The sense of smell. Annual Review of Psychology, $1973,24,187-206$.

Ekman, G., Berglund, B., Berglund, U., \& Lindvall, T. Perceived intensity of odor as a function of time of adaptation. Scandinavian Journal of Psychology, 1967, 8, 177-186.

Indow, T., \& Stevens, S. S. Scaling of saturation and hue. Perception \& Psychophysics, 1966, 1, 253-271.

LiNDVALL, T. On sensory evaluation of odorous air pollutant intensities. Nordisk Hygienisk Tidskrift, 1970, Supplement 2, $1-181$.

Lindvall, T., \& SVEnsson, L. T. Equal-unpleasantness matching of malodorous substances in the community. Journal of Applied Psychology, 1974, 59, 264-269.

Le Magnen, I. Nouvelles données sur le phenomène de l'exaltoxide. Comptes Rendus Académie des Sciences (Paris), 1950, 230, 1103-1105.

Stevens, S. S. On the new psychophysics. Scandinavian Journal of Psychology, 1960, 1, 27-35.

Stevens, S. S. Power-group transformations under glare, masking, and recruitment. Journal of the Acoustical Society of America, 1966, 39, 725-735.

Stevens, S. S. Tactile vibration: Changes of exponent with frequency. Perception \& Psychophysics, 1968, 3, 223-228.

Stevens, S. S., \& Greenbaum, H. M. Regression effects in psychophysical judgement. Perception \& Psychophysics, 1966, 1, 439-446.

Svensson, L. T., \& Szczygiel, K. A matching method of successive approximations and its instrumentation. Behavior Research Methods \& Instrumentation, 1974, 6, 13-18.

(Received for publication January 14, 1974; accepted February 21, 1974.) 